\title{
Evaluation of Surgical Outcomes, Patient Satisfaction, and Potential Complications after Blepharoplasty
}

\author{
(D) Sezen Akkaya \\ Department of Ophthalmology, Fatih Sultan Mehmet Training and Research Hospital, Istanbul,Turkey
}

\begin{abstract}
Objectives: This study is an evaluation of the surgical outcomes, patient satisfaction, and potential complications following blepharoplasty surgery.

Methods: A total of 128 patients who underwent blepharoplasty surgery between 2015 and 2017 were included in this study. A postoperative lid crease difference of at least $1 \mathrm{~mm}$ was considered asymmetrical. Pre- and postoperative tear break-up time (TBUT) was evaluated. All of the patients were asked to complete a satisfaction assessment form prepared by the authors. The scar tissue that developed along the incision line was evaluated and graded by a single observer.

Results: The mean age of the patients was $62.70 \pm 9.73$ years, and all of the patients were monitored for a period of least 6 months. In combination with blepharoplasty, 17 patients underwent eyebrow surgery, 6 underwent lower eyelid surgery, and 4 underwent levator surgery. Lid crease asymmetry of $1 \mathrm{~mm}$ was determined in 8 patients, and a 2-mm asymmetry was identified in 2 patients, although the pre- and postoperative TBUT was not significantly different $(p \geq 0.05)$. A total of $107(83.59 \%)$ patients reported a very high level of satisfaction at the postoperative sixth month, I5 (II.7I\%) patients reported a high level of satisfaction, and $6(4.68 \%)$ reported a moderate level of satisfaction. In the postoperative sixth month, $87(67.97 \%)$ patients had no scar tissue at the incision line, while $36(28.13 \%)$ patients had noticeable scar tissue upon close inspection, and $5(3.91 \%)$ patients had apparent scar tissue. None of the patients developed serious complications, such as severe hematoma, lagophthalmos, or wound separation.

Conclusion: In the present study, the patients generally expressed long-term satisfaction with the results of blepharoplasty surgery for dermatochalasis. A comprehensive preoperative assessment, and combining surgeries when necessary and appropriate, ensures a high rate of success in blepharoplasty procedures.

Keywords: Blepharoplasty, patient satisfaction, potential complications.
\end{abstract}

\section{Introduction}

Blepharoplasty surgery is performed to correct changes to the eyelid that may be caused by aging or genetic disposition. Among middle-aged and elderly individuals, the removal of the orbicular muscle and fat tissue during an upper eyelid blepharoplasty provides a younger look and increases the field of vision, particularly in the upper temporal region (I). Surgical success depends primarily on meeting the patients' expectations and achieving a uniform, symmetrical appearance after the operation (2). An eyebrow elevation during blepharoplasty may also be appropriate in cases where dermatochalasis is accompanied by eyebrow ptosis, as it may negatively affect the cosmetic outcome by reducing the distance between the eyebrow and the eyelid (3). The surgeon should also be able to manage potential postoperative complications, such as lagophthalmos, asymmetry, and eyebrow ptosis (4). While blepharoplasty surgery is often successful, rarely, complications may arise. The frequency of dry eye after blepharoplasty is higher among individuals with preoperative tear insufficiency, but it may also develop due to insufficient postoperative blinks and lagophthalmos. In previous studies, the reported frequency of dry eye following blepharoplasty ranged between $8 \%$ and $21 \%(5,6)$. Aside from the possible negative cosmetic outcomes, such as eyelid

Address for correspondence: Sezen Akkaya, MD. Department of Ophthalmology, Fatih Sultan Mehmet Training and

Research Hospital,Istanbul,Turkey

Phone: +90 2165783000 E-mail: drsezenakkaya@gmail.com

Submitted Date: April 15, 2018 Accepted Date: June 19, 2018 Available Online Date: July 10, 2018

${ }^{\circ}$ Copyright 2018 by Beyoglu Eye Training and Research Hospital - Available online at www.beyoglueye.com 
asymmetry, lagophthalmos, or changes in facial expression, complications such as dry eye syndrome and superior limbic keratoconjunctivitis may also emerge after blepharoplasty, and retro-orbital bleeding is a potential complication that may, in rare cases, result in vision loss (7-II).

In the present study, the long-term outcomes regarding scar development, asymmetry, findings of dry eye, and the level of satisfaction among patients who underwent blepharoplasty surgery were evaluated.

\section{Methods}

The study included a total of 128 patients who underwent upper eyelid blepharoplasty surgery at least 6 months earlier due to ptosis associated with a decreased visual field. Informed consent forms were obtained from all of the patients prior to their inclusion in the study. The pre- and postoperative examinations of all of the patients were carried out by the same physician, and tear break-up time (TBUT) was recorded. The need to perform simply upper eyelid or also lower eyelid surgery, and internal-external eyebrow surgery and correction for ptosis were all evaluated according to the specific problems of each patient. The staging of the scar tissue along the incision line was performed in the postoperative sixth month, in accordance with the following stage definitions, 0: no scar tissue along the incision line, I: scar tissue can be seen upon close inspection, 2: scar tissue is apparent, 3: marked hypertrophic scar.

Before administering a local anesthetic, the surgical site was marked on the patients while they were sitting up straight on the operating table. In patients who had a visible lid crease, the first line of incision was the lid crease. In patients who lacked visible a lid crease, the eyelid was turned out, and the line corresponding to the upper boundary of the tarsal plate was used as the first line of incision. Excess skin tissue was determined with a pair of forceps, and care was taken to leave at least 22 to $24 \mathrm{~mm}$ of tissue, in total, on the upper part of the excised region toward the lower boundary of the eyebrow and the lower part to the eyelid boundary. To ensure that the eyes were symmetrical, the distance between the inner parts of the eyebrow, the middle highest part of the eyebrow, and the end of the eyebrow and the lid creases (estimated saturation line) were measured and equalized.

A local anesthetic ( $50 \%$ lidocaine [with adrenaline], $50 \%$ bupivacaine mixture) was administered subcutaneously using a dental needle. The skin on the marked line was incised using a number-II surgical scalpel blade to the level of the orbicular muscle, and both the skin and the muscle were excised simultaneously. Hemostasis was achieved through unipolar/bipolar cauterization. Pressure was applied to the globe to check for fat tissue prolapsus, and when necessary, the septum was opened to clamp and excise fat tissue, after which hemostasis was achieved through cauterization. To form the lid crease, 6-0 Prolene (Ethicon, Inc., Somerville, NJ, USA) sutures were used through skin-tarsus-orbicular muscle-skin lines, with 3 vertical sutures applied in women, and 2 in men. The skin was sutured using 6-0 Prolene sutures while the wound lips were kept opposite.

All of the patients were prescribed an antibiotic ointment to be used postoperatively twice-daily, and all of the sutures were removed on the 10th day of follow-up. The patients were, at a minimum, followed-up in the first, third, and sixth postoperative months. The patients were asked to complete a satisfaction assessment form that comprised 3 yes-no questions.

The patients were asked to classify their postoperative level of satisfaction as 0: I am not satisfied, I: I am moderately satisfied, 2: I am satisfied, 3: I am very satisfied, and I would recommend it to another.

Statistical analyses of the mean values were performed using Statistics for Windows, Version 18.0 (SPSS, Inc., Chicago, IL, USA). A p value that was $\leq 0.05$ was considered statistically significant.

\section{Results}

A total of 128 patients with a mean age of $62.70 \pm 9.73$ years were included in the study. Among the patients, 118 (92.1\%) were women, while $10(7.8 \%)$ were men. The mean duration of follow-up was $12.9 \pm 5.6$ months.

In combination with blepharoplasty, 17 patients underwent eyebrow surgery, 6 underwent lower eyelid surgery, and 4 underwent levator surgery. In all, II patients underwent combined internal eyebrow surgery, while 6 underwent external eyebrow surgery.

When the degree of asymmetry was assessed, a I-mm lid crease asymmetry was identified in 8 patients, and a 2-mm asymmetry was observed in 2 patients. One patient underwent repeat surgery due to asymmetry, which was corrected with no scar development.

Table I illustrates the postoperative satisfaction assessment form. In the late postoperative period, $6(4.68 \%)$ pa-

Table I. Post-operative satisfaction assessment form and results Yes (\%)

Are you satisfied with the results of your operation?

$$
\begin{aligned}
& \text { Very good } \\
& \text { Good }
\end{aligned}
$$

Moderate

4.68

Would you have the same surgery again, knowing

95

what it involves?

Do you feel more energetic and awake during the 89 


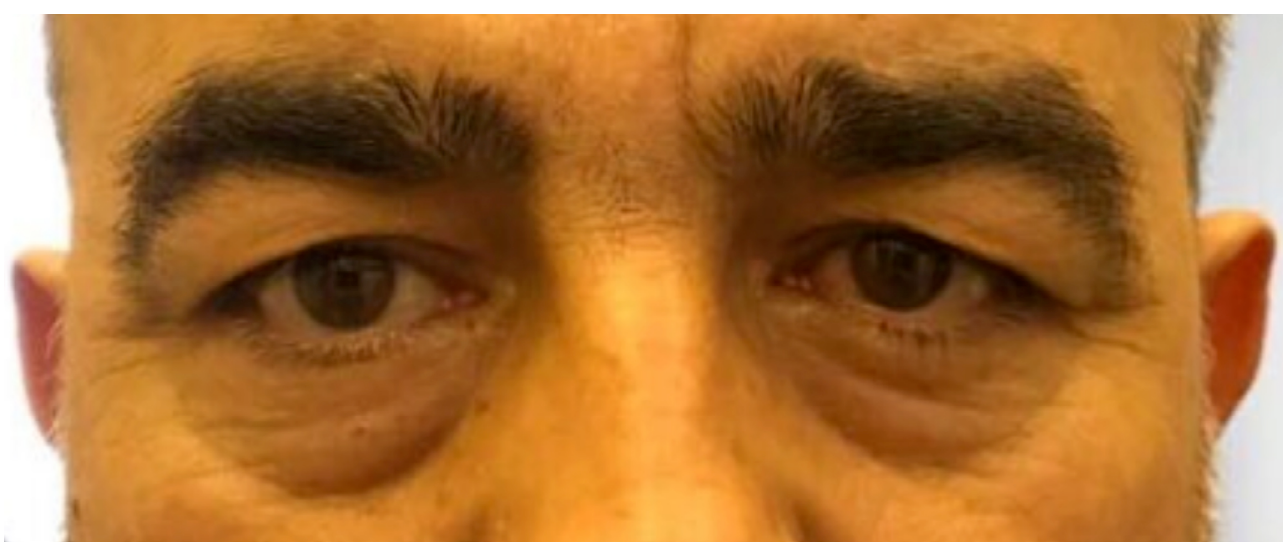

Figure I. A preoperative photo of a patient.

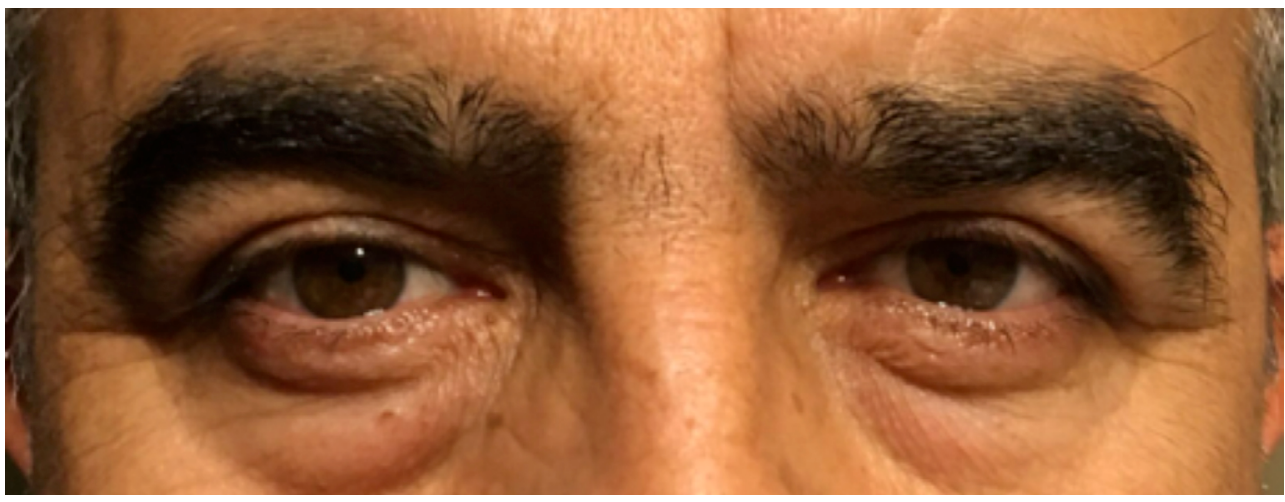

Figure 2. A postoperative photo of the same patient.



Figure 3. Scar tissue visible on close inspection.

tients reported being "moderately satisfied," I5 (I I.72\%) reported being "satisfied," and 107 (83.59\%) reported being "very satisfied" with the surgery, while none responded that they were not satisfied. Of the 6 patients who reported being moderately satisfied, the cause of discomfort was asymmetry in 3 of the patients, and the appearance of scar tissue in the remaining 3 patients. One patient with asymmetry was re-operated on, and symmetry was achieved. A second surgery was not deemed necessary for the remaining 2 patients.

Figures $I$ and 2 are photographs of a patient before and after surgery. He reported being very satisfied with the results.

In the late postoperative period, 87 (67.97\%) patients had no scar tissue on the incision line, 36 (28.13\%) had some scar tissue that was noticeable upon close inspection, and $5(3.91 \%)$ had apparent scar tissue. None of the patients had marked hypertrophic scar tissue (Table 2). Figure 3 is a photograph of a patient with scar tissue that was noticeable upon close inspection. Figure 4 is a patient photograph demonstrating apparent scar tissue.

The mean preoperative and postoperative TBUT was I3.48 \pm 2.4 seconds and $12.5 \pm 1.9$ seconds, respectively. The difference was not statistically significant ( $p>0.05)$. Among the patients, 19 (14.8\%) developed milia along the wound boundaries during the early postoperative term. 
Table 2. Post operation scar staging and results

\begin{tabular}{lc} 
Scar stage & Patients (\%) \\
\hline 0: No scar tissue along the incision line & $87(67.97)$ \\
I: Scar tissue can be seen upon close inspection & $36(28.13)$ \\
2: Scar tissue is apparent & $5(3.91)$ \\
3: Marked hypertrophic scar & $0(0)$ \\
\hline
\end{tabular}

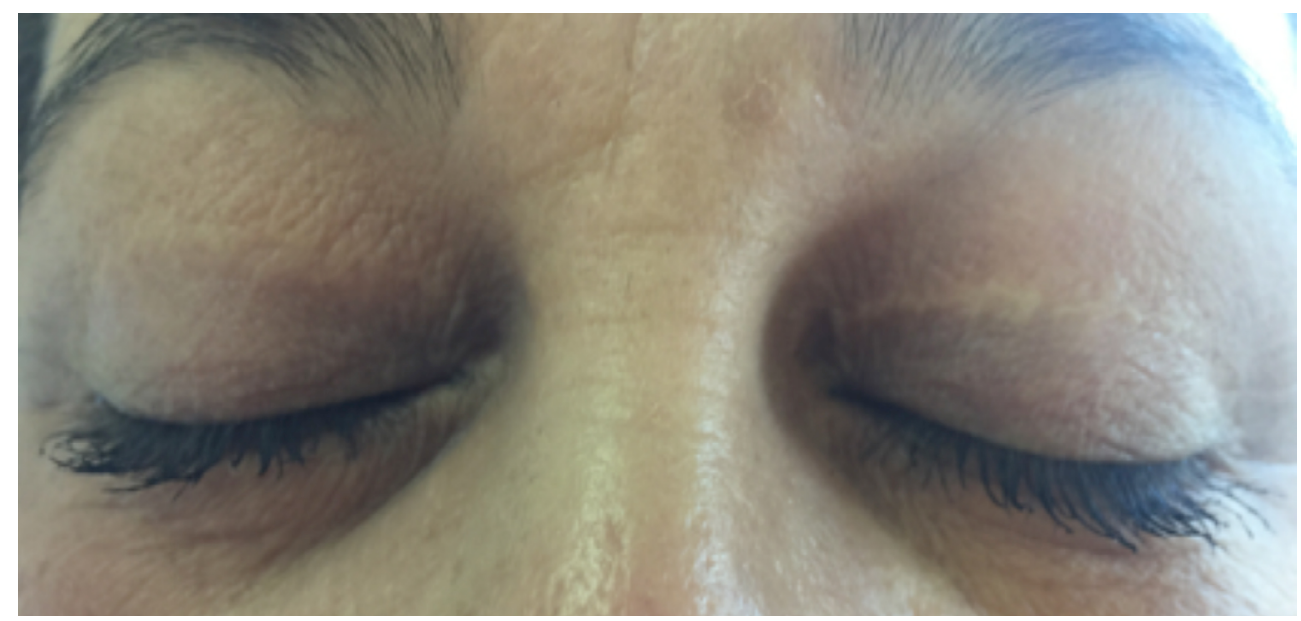

Figure 4. Visible scar tissue.

\section{Discussion}

While blepharoplasty is a surgical intervention with a high success rate, it is still essential to perform a detailed examination of the patient before surgery and to devise a sound surgical plan in advance. The most common complications associated with blepharoplasty include asymmetry, edema, infection, lagophthalmos, and secondary keratopathy, canthal folds, and ectropion. While these complications can be treated, loss of vision is still a very serious complication that has been reported in the literature at a rate of $0.004 \%$ ( II, 12). These severe complications are more common after lower eyelid blepharoplasty operations, and are seen only rarely following upper eyelid blepharoplasty.

Although a symmetrical appearance was achieved in 92.19\% of the patients operated on in our clinic, asymmetry was still the most commonly encountered complication. A total of 10 patients had lid crease asymmetry: there was a difference of $2 \mathrm{~mm}$ in 2 patients, but only I required a second operation. The remaining patients did not undergo additional interventions, given that they had no complaints and did not elect to have a second surgery.

Following blepharoplasty, dry eye syndrome develops in $8 \%$ to $21 \%$ of patients $(6,13)$, and lower eyelid malpositioning, increased eyelid space, lagophthalmos, eyelid retraction, and decreased blink reflex may play a role in its development (14). Previous studies have indicated that the symptoms associated with dry eye syndrome develop more frequently after lower eyelid surgeries. In their study that reported the outcomes of blepharoplasty surgery in 892 patients, Prischmann et al. (I3) reported a rate of postoperative dry eye of $12.9 \%$ of the patients who underwent upper eyelid blepharoplasty alone, and $21.9 \%$ in patients who underwent lower eyelid surgery.

In the present study, the rate of postoperative dry eye syndrome requiring treatment did not significantly increase, and there was no significant postoperative change in TBUT. This was probably due to the fact that our patients were not operated on for cosmetic reasons, but required surgery due to dermatochalasis limiting the field of vision. In addition, we made only limited skin excisions in all cases. The need to use artificial tears was greater during the postoperative first due to limited eyelid movement during this period, but this returned to normal after the postoperative I0th day. To prevent the development of postoperative dry eye, a serious potential complication of surgery, the decision to operate must be considered very carefully in patients with severe dry eye syndrome; and most importantly, excisions must be limited in order to avoid lagophthalmos.

Achieving a symmetrical appearance after surgery depends on adequate evaluation of the preoperative eyebrow position, the eyebrow-eyelash edge distance, the appearance of the tarsal plate, and the eyelid edge-corneal reflex distance. Among these parameters, the amount of tarsal plate contributes most to symmetry, and is the most no- 
table element with respect to appearance. For this reason, in patients with unnoticeable lid creases, it is crucial that the eyelid is turned out preoperatively and that a lid crease is formed symmetrically between the 2 eyes based on the upper boundary of the tarsal plate.

Although a visible incision site tends to be less frequent after upper eyelid blepharoplasty procedures, patient satisfaction is still linked to the absence of incision marks. To reduce the appearance of scars, the wound edges should be uniformly and smoothly joined while suturing, the sutures should not be too tight, and the minimal number of lines possible should be used. In patients who will undergo concomitant eyebrow surgery, it is important to avoid extra incisions and to pay attention to achieving symmetry. As ectropion is far more frequent in lower eyelid blepharoplasties, it is essential that preoperative measurements and marks be made very carefully before these procedures.

A similar study was previously conducted by Schulz et al. (15), who reported the results of blepharoplasty in 47 patients and identified a surgery success rate of $91.5 \%$ in terms of symmetry. The symmetry success rate in the present study was similar at $\mathbf{9 2 . 1 9 \%}$. Schulz et al. identified a patient satisfaction ratio of $95.7 \%$, and the $95.31 \%$ total ratio of satisfied and highly satisfied patients in the present study was consistent with those results. Schulz et al. also assessed patient quality of life, and found that it increased after blepharoplasty.

Lèclere et al. (16) published the asymmetry ratio identified in laser-assisted blepharoplasty performed on 52 patients, and they determined that 8 patients had $1 \mathrm{~mm}$ of asymmetry. In all, 6 were re-operated on. Leclere et al. had a comparatively higher ratio of asymmetry.

In this study, the patients expressed long-term satisfaction with the outcome of blepharoplasty surgery performed due to dermatochalasis. A comprehensive preoperative assessment and combination surgery when appropriate can ensure a high rate of success in blepharoplasty procedures.

\section{Disclosures}

Peer-review: Externally peer-reviewed.

Conflict of Interest: None declared.

\section{References}

I. Lee JW, Baker SR. Esthetic enhancements in upper blepharoplasty. Clin Plast Surg 2013:40;139-46. [CrossRef]

2. Yang P, Ko AC, Kikkawa DO, Korn BS. Upper Eyelid Blepharo- plasty: Evaluation, Treatment, and Complication Minimization. World J Plast Surg 2016;5:58-6I.

3. Hassanpour SE, Khajouei Kermani H. Brow Ptosis after Upper Blepharoplasty: Findings in 70 Patients. Semin Plast Surg 2017;31:5|-7.

4. Leatherbarrow B, Saha K. Complications of Blepharoplasty. Facial Plast Surg 2013;29:28I-8. [CrossRef]

5. Hamawy AH, Farkas JP, Fagien S, Rohrich RJ. Preventing and managing dry eyes after periorbital surgery: a retrospective review. Plast Reconstr Surg 2009;123:353-3. [CrossRef]

6. Floegel I, Horworth-Winter J, Mueller K, Haller-Schober EM. A conservative blepharoplasty may be a means of alleviating dry eye symptoms. Acta Ophthalmol Scand 2003;81:230-2.

7. Witney $\mathrm{S}$, Witherow $\mathrm{H}$, Waterhouse. One hundred cases of endoscopic brow lift. Br J Plast Surg 2002;55:20-4. [CrossRef]

8. Jones BM, Grover R. Endoscopic brow lift: a personal review of 538 patients and comparison of fixation tecniques. Plast Reconstr Surg 2004; I 1 3:1242-50. [CrossRef]

9. Saadat D, Dresner SC. Safety of blepharoplasty in patients with preoperative dry eyes. Arch Facial Plast Surg 2004;6:10 I-4.

10. Sheu MC, Schoenfield L, Jeng BH. Development of superior limbic keratoconjunctivitis after upper eyelid blepharoplasty surgery: support for the mechanical theory of its pathogenesis. Cornea 2007; 26:490-2. [CrossRef]

II. Hass AN, Penne RB, Stefanyszyn MA, Flanagan JC. Incidence of postblepharoplasty orbital hemorrhage and associated visual loss. Ophthal Plast Reconstr Surg 2004;20:426-32. [CrossRef]

12. Patrocinio TG, Loredo BA, Arevalo CE, Patrocinio LG, Patrocinio JA. Complications in blepharoplasty: how to avoid and manage them. Braz J Otorhinolaryngol 201 I;77:322-7. [CrossRef]

13. Prischmann J, Sufyan A, Ting JY, Ruffin C, Perkins SW. Dry eye symptoms and chemosis following blepharoplasty: a 10-year retrospective review of 892 cases in a single-surgeon series. JAMA Facial Plast Surg 2013;15:39-46. [CrossRef]

14. Korn BS, Kikkawa DO, Schanzlin DJ. Blepharoplasty in the postlaser in situ keratomileusis patient: preoperative considerations to avoid dry eye syndrome. Plast Reconstr Surg 2007; I 19:22329. [CrossRef]

15. Schulz CB, Nicholson R, Penwarden A, Parkin B. Anterior approach white line advancement: technique and long-term outcomes in the correction of blepharoptosis. Eye (Lond) 2017;31:1716-23. [CrossRef]

16. Leclère FM, Alcolea J, Mordon S, Servell P, Kolb F, Unglaub F, et al. Long-term outcomes of laser assisted blepharoplasty for ptosis: about 104 procedures in 52 patients. J Cosmet Laser Ther 2013;15:193-9. [CrossRef] 